\title{
Abstracts - eine facettenreiche Textsorte der Wissenschaft
}

\author{
Ines Busch-Lauer (Zwickau)
}

\begin{abstract}
Der Beitrag beschreibt die Relevanz der informationsverdichtenden Textsorte Abstract in der Wissenschaftskommunikation. Im Mittelpunkt stehen die Definition, die Klassifikation und die Struktur sowie ausgewählte Merkmale dieser Textsorte.

Im ersten Teil des Beitrags werden die unterschiedlichen Arten von Abstracts anhand von Textbeispielen aus der Linguistik, der Medizin und den Technikwissenschaften expliziert. Im zweiten Teil untersucht der Beitrag anhand von Abstracts, die von deutschen Studierenden der Technik- und Ingenieurwissenschaften im Rahmen ihrer fachbezogenen Englischausbildung verfasst wurden, inwieweit die textsortenimmanenten Merkmale auch von Lernenden in der Textproduktion in der Fremdsprache umgesetzt wurden. Mit dieser qualitativ beschreibenden Untersuchung trägt die Studie zur kontrastiven Fachtextsortenbeschreibung und andererseits als Praxisbericht zur Vermittlung von Textsorten im fachbezogenen Fremdsprachenunterricht an Hochschulen bei.
\end{abstract}

\section{$1 \quad$ Abstracts in der Fachkommunikation}

\subsection{Ziel und Gegenstand des Beitrags}

Versucht man sich heute über ein Sachthema zu informieren, dann ist dies angesichts der Informationsflut ohne Rückgriff auf Suchmaschinen im Internet oder auf Index- und Abstractverzeichnisse in Bibliotheken und die gezielte Quellenselektion fast nicht $\mathrm{zu}$ bewerkstelligen. Spezialisierungs- und parallel dazu verlaufende Globalisierungsprozesse produzieren in den verschiedenen Wissenschaftsdisziplinen immer mehr Wissen - die Zahl fachspezifischer, fächerübergreifender und popularisierender Publikationen wächst. Der Wettbewerb um neue wissenschaftliche Erkenntnisse führt zudem zu einem enormen Publikationsdruck für Fachwissenschaftler und den wissenschaftlichen Nachwuchs, da ihre Leistungsfähigkeit oft genug an der Zahl der in renommierten Fachorganen erschienenen Publikationen gemessen wird. In diesem Zusammenhang gewinnen auch in Englisch publizierte Beiträge enorm an Bedeutung. Wir sind folglich mit einer zunehmenden, sich sprachlich, aber auch medial verändernden Informationswelle konfrontiert. Dies hat insbesondere Textsorten, die Informationen komprimiert darstellen bzw. miteinander verlinken, in das Blickfeld linguistischer Forschung gerückt. Das Abstract wurde u. a. in den folgenden Arbeiten analysiert bzw. beschrieben: Baßler (2003); Dahl (2009); Dayrell (2009); Feak/Swales (2009); Fluck (1988, 1989); Gläser (1990, 1991); Gnutzmann (1991); Golebiowski (2009); Graetz (1985); Lorés-Sanz (2009); Maher (1990); Martín-Martín (2005); McIntosh (1996), Melander et al. (1994, 1997); Salager-Meyer (1990, 1992); Swales (1990); Tsai (2010); Ventola (1994). Abstracts werden inzwischen auch in vielen gängigen Publikationen zum wissenschaftlichen Schreiben angesprochen, allerdings gibt es darin kaum konkrete Hinweise zu ihren textuellen Merkmalen bzw. zu Unterschieden zwischen Wissenschaftsdisziplinen und Sprachen (vgl. für das Englische z.B. Cargill/O'Connor 2009; 
Feak/Swales 2009; Skern 2009). Daneben hat die Textsorte Abstract in ihrer informationskomprimierenden Ausprägung auch das besondere Interesse von Dokumentationswissenschaftlern geweckt.

Ziel des vorliegenden Beitrages ist es, die Textsorte Abstract in Bezug auf ihren Facettenreichtum innerhalb verschiedener Disziplinen und ihrer sprachlichen Merkmale vorzustellen. Im ersten Teil des Beitrags werden die unterschiedlichen Arten von Abstracts anhand von Textbeispielen aus der Linguistik, der Medizin und den Technikwissenschaften expliziert. Im zweiten Teil untersucht der Beitrag anhand von Abstracts, die von deutschen Studierenden der Technik- und Ingenieurwissenschaften im Rahmen ihrer fachbezogenen Englischausbildung verfasst wurden, inwieweit die Textsortenmerkmale auch von Lernenden in der Textproduktion in der Fremdsprache umgesetzt wurden. Mit dieser qualitativen Betrachtung soll die Studie zur Textsortenbeschreibung beitragen und andererseits als Praxisbericht zur Vermittlung von Textsorten im fachbezogenen Fremdsprachenunterricht an Hochschulen dienen.

\subsection{Zur kommunikativen Funktion von Abstracts}

Wissenschaftler, aber auch Fachleute in Wirtschaft und Technik lesen Abstracts (dt. auch Kurzreferate), um einen Überblick über aktuelle Publikationen in ihrem Fachgebiet bzw. zu einem bestimmten Thema zu erhalten. Sie produzieren Abstracts, um dem Fachpublikum neue Fachinhalte über etablierte Referateorgane (engl. Abstracting Journals) schnell verfügbar zu machen und so die themenbezogene Selektion von Fachartikeln zu erleichtern. Der Titel eines Dokumentes, die Schlüsselwörter und das Abstract sind daher zu entscheidenden Informationsquellen für eine optimale Informationsrecherche in Bibliotheken und Wissensdatenbanken geworden. Aber nicht nur Fachleute, sondern auch Studierende und Nichtfachleute (z. B. Fachsprachenlehrer und Übersetzer) können aus der Rezeption von Abstracts Nutzen ziehen, da diese Kurztexte in den Referateorganen nach der Fachgebietsstruktur oder thematisch geordnet sind. Sie bieten auf diese Weise eine Orientierung zur Fachgebietsstruktur und -terminologie.

\section{Abstracts in der Wissenschaftskommunikation}

\subsection{Zur Definition und Klassifikation von Abstracts}

Ein Kurzreferat ist die nicht wertende Angabe des für Informations- und Dokumentationszwecke wesentlichen Inhaltes eines Dokuments, die das Ziel hat, dem Benutzer die Beurteilung der Relevanz des Dokuments zu erleichtern (KTS 1975: 88; vgl. auch ISOStandard 214-1976 (E) bzw. DIN-Norm 1426, 188: 2-3).

Mit dieser Definition gehört das Abstract zunächst zur Kategorie der Inhaltsangaben, wie z. B. Inhaltsverzeichnis (stichpunktartige Liste der Inhalte eines Dokuments, das vor dem Text platziert wird; Annotation (wertende Inhaltsdarstellung) und Rezension (Buchbesprechung, in der die Inhalte eines Buches durch einen fremden Autor einer kritischen Analyse unterzogen werden). Im Unterschied zu diesen Textarten sind Abstracts wertneutrale, inhaltlich und formal abgegrenzte, meist von einem Ausgangstext abgeleitete Kurztexte im Umfang von bis zu 250 Wörtern, die zusammen mit dem Ausgangstext (Primärtext) oder räumlich und zeitlich davon getrennt publiziert werden.

In den Untersuchungen von Adolphi (1996), Gläser (1990: 117) und Swales/Feak (1994: 210) wird die Textsorte Abstract als Sekundärtextsorte/abgeleitete Textsorte betrachtet und als Verallgemeinerung aus den drei Textsortenvarianten: (1) Konferenzabstract (Gläser 1990: 50f.), (2) Abstract wissenschaftlicher Zeitschriftenaufsätze; (3) Abstract in einem Referateorgan (Gläser 1990: 117). 
Unter kommunikativen Gesichtspunkten lassen sich die folgenden Typen von Abstracts unterscheiden:

(1) Nach dem Zeitpunkt der Texterstellung unterscheidet man zwischen retrospektiven und prospektiven Abstracts (Prätext). Retrospektiv bedeutet, dass das Abstract nach der Produktion des Primärtextes entstanden ist (z. B. das Abstract eines wissenschaftlichen Zeitschriftenaufsatzes). Prospektiv bedeutet, dass zunächst ein Abstract verfasst wird und der Primärtext erst später entsteht (z. B. Abstract für einen Konferenzbeitrag). Das retrospektiv bzw. prospektiv ausgerichtete Extended Abstract und Executive Summary wird in der Wirtschaft oder in den Naturwissenschaften für umfangreiche Dokumente genutzt und publiziert den Inhalt eines Textes im Umfang von 2-5 Druckseiten an Stelle des kompletten Originals, z.B. im Internet (vgl. Adolphi 1996).

(2) Nach dem Verfasser des Kurztextes wird zwischen Autorenabstract, Fremdabstract und maschinell erstelltem Abstract unterschieden. Das Autorenabstract wird vom Autor des Primärtextes formuliert. Beim Fremdabstract übernimmt häufig ein Dokumentationswissenschaftler das Verfassen des Textes nach einheitlichen Vorgaben für ein Referateorgan. Darüber hinaus werden für die Erstellung von Fremdabstracts aufgrund der Informationsflut auch IT-gestützte Systeme zur Extraktion der wichtigsten Inhaltselemente (Makropropositionen) genutzt. Diese Schlagwortabstracts gehören in die Gruppe der maschinell erstellten Abstracts.

(3) Nach dem inhaltlichen Bezug wird zwischen indikativen, informativen und aus beiden Formen gemischten Abstracts unterschieden.

(4) Darüber hinaus können Abstracts auch nach ihrer Platzierung im Text klassifiziert werden: (1) als Paratext (im Primärtext zwischen Titel und Volltext), (2) am Ende einer Zeitschrift/eines Sammelbandes (z.B. als Abstract in der Fremdsprache) oder (3) isoliert in einem Abstracting Journal (vgl. Baßler 2000).

(5) Nach Form und Layout unterscheidet man zwischen Text-, Struktur- und Schlagwortabstracts. Die für die meisten Wissenschaftsdisziplinen typischen Texte sind ausformulierte Abstracts. Daneben haben sich besonders in der Medizin und der Technik auch Strukturabstracts etabliert. Zur Erstellung dieser Texte nutzt der Autor die von einem Publikationsorgan standardisiert vorgegebenen Textelemente, die oft dem Aufbau eines wissenschaftlichen Aufsatzes/eines Experimentes folgen, (z.B. IMRADStruktur in der Forschung oder z. B. in der Medizin: Einleitung, Patienten und Methode, Ergebnisse, Schlussfolgerungen). Diese Bausteine werden durch in kurzen Sätzen/als Phrasen formulierte Inhaltselemente aus dem Dokument zu einem strukturierten Text ergänzt. In der Medizin ist dieser Abstract-Typ in allen internationalen Publikationsorganen Standard und vereinfacht die Informationserfassung und verarbeitung. Die dritte Kategorie, das Schlagwortabstract, basiert auf Kernbegriffen (engl. keywords) eines Dokumentes und gibt den Inhalt stichpunktartig wieder.

(6) Im Zuge der Globalisierung sind die meisten internationalen wissenschaftlichen Publikationsorgane zur mehrsprachigen Publikation von Abstracts übergegangen. Neben der Publikationssprache eines Dokumentes nutzt man Englisch bzw. Französisch oder Spanisch, so dass zwischen Abstracts in der Dokumentsprache und in einer Fremdsprache unterschieden werden kann. Häufig ist die Qualität der übersetzten Abstracts hinsichtlich sprachlich-kultureller Aspekte jedoch nicht kommunikativ adäquat.

Dieser grobe Überblick zur Definition und Klassifikation von Abstracts zeigt, dass diese Textsorte durch das überschaubare Textformat eine effektive Möglichkeit bietet, Informationen aufzubereiten, zu speichern und weiterführend zu verarbeiten. Abstracts dienen 
folglich der Informationsrecherche, aber auch der Speicherung von Informationen in Wissensdatenbanken. Durch ihre terminologische Dichte eignen sie sich sehr gut für die Erarbeitung von Fachwortschatz im fachbezogenen Fremdsprachenunterrichts.

\subsection{Beispiele für die Klassifikation von Abstracts nach dem inhaltlichen Bezug}

Für das bessere Verständnis der Textsorte Abstract und zu ihrer Vermittlung im fachbezogenen Sprachunterricht betrachten wir im Folgenden die in der DIN-1426 (1988: 3) benutzte Klassifizierung nach dem inhaltlichen Bezug: (1) indikatives, (2) informatives und (3) informativ-indikatives Abstract (Referat) sowie (4) Strukturabstract. Je ein Beispiel veranschaulicht die unterschiedlichen Typen:

Das indikative Referat gibt lediglich an, wovon ein Dokument handelt. Es weist den Leser auf die im Dokument behandelten Sachverhalte hin und deutet die Art der Behandlung an, aber gibt nicht konkrete Resultate der im Dokument enthaltenen Überlegungen oder dargestellten Untersuchungen wieder. (Quelle: DIN-1426 1988: 3)

Indikative Abstracts sind in Textform verfasst, sie werden häufig zwischen dem Titel und dem Primärtext eines Dokumentes abgedruckt. In der Regel sind indikative Abstracts Autorenabstracts.

Beispiel 1 ist typisch für ein indikatives Abstract, wie es in den Geisteswissenschaften zu finden ist. Die Sätze wurden durchnummeriert, die wesentlichen Textbausteine zur Veranschaulichung mit Fettdruck markiert und die damit verfolgten Ziele in Klammern erfasst.

\section{Beispiel (1):}

(1) Als Ergebnis der interdisziplinären Öffnung der Fachsprachenforschung hat sich die Untersuchung der Beziehung zwischen Sprache und Denken in ihrer spezifischen Ausprägung als Beziehung von Fachsprache und Fachdenken zu einer aktuellen Herausforderung der Fachsprachenforschung entwickelt. (2) Der vorliegende Beitrag basiert auf einer kürzlich an der Philologischen Fakultät der Universität Leipzig eingereichten Dissertation. (Sätze 1 und 2: ABLEITUNG EINER FORSCHUNGSAUFGABE/EINORDNUNG DES THEMAS) (3) Das Hauptziel bestand darin (ZIELANGABE), anhand ausgewählter Analysekategorien auf verschiedenen Fachtextebenen zu untersuchen, wie sich der Einfluss des technikwissenschaftlichen Fachdenkens in der Gestaltung von Fachzeitschriftenartikeln aus dem Kommunikationsbereich Werkstoffwissenschaft manifestiert. (HINWEIS AUF METHODE) (4) Im vorliegenden Beitrag steht die rhetorisch- stilistische Fachtextdimension am Beispiel der Verwendung von Heckenausdrücken (Hedges) im Mittelpunkt (PRÄZISIERUNG DES UNTERSUCHUNGSGEGENSTANDES). (5) Nach der Beschreibung des methodischen Vorgehens werden die Ergebnisse der Untersuchung eines repräsentativen Korpus werkstoffwissenschaftlicher Zeitschriftenartikel u.a. in ihrer Relation zur Spezifik der technikwissenschaftlichen Erkenntnisprozesse vorgestellt (INHALTLICHE GLIEDERUNG DES ARTIKELS). (Quelle: Fachsprache, 3-4/2007: 152, Hervorhebungen durch IBL)

Im Gegensatz zum beschreibenden indikativen Abstract, werden in einem informativen Abstract die wichtigsten Inhaltselemente eines Dokumentes verarbeitet. Auf metakommunikative Elemente wird verzichtet.

Das informative Kurzreferat gibt so viel Information wieder wie Typ und Stil des Dokumentes zulassen. Es gibt insbesondere Auskunft über das behandelte Gebiet, Zielsetzungen, Hypothesen, Methoden, Ergebnisse und Schlussfolgerungen der im Originaldokument enthaltenen Überlegungen und Darstellungen, einschließlich der Fakten und Daten. (Quelle: DIN-1426 1988: 3)

Das aus dem Bereich Technik entnommene Beispiel 2 kann als informatives Abstract klassifiziert werden, da es genau beschreibt, was untersucht wurde und welche Ergebnisse 
dabei festzustellen waren. Typisch für diesen komprimierten deutschen Wissenschaftstext sind der Nominalstil, die Verwendung von Passivkonstruktionen und der hohe Anteil von Fachlexik zur Beschreibung des Verfahrens und der Ergebnisse.

Beispiel (2):

(1) Um Bauteile gegen grob abrasiven Verschleiß zu ertüchtigen, besteht die Möglichkeit der Aufbringung dicker verschleißbeständiger Schichten, beispielsweise durch Auftragschweißen, thermisches Spritzen oder Heiß-Isostatisches Pressen von Verbunden (HIP-Cladding) (THEMENEINGRENZUNG).

Mit "Sintercladding" wurde nun ein Verfahren entwickelt, dass den wesentlichen Vorteil des HIP-Cladding, mehrere Millimeter dicke, rissfreie Schichten zu erzeugen, zu wesentlich geringeren Kosten erlaubt (VERFAHRENSNENNUNG; VORTEILSANGABE). Das Verfahren basiert im Wesentlichen auf pulverförmigen Ausgangswerkstoffen aus Werkzeugstahl und Keramik, die in einem Vakuumsinterprozess mit kurzen Prozesszeiten verdichtet und dabei auf das zu beschichtende Bauteil aufgebracht werden können (VERFAHRENSBESCHREIBUNG). Die resultierenden verschleißbeständigen Schichten weisen Eigenschaften auf, die denen vergleichbarer HIP-Schichten ebenbürdig (sic!) sind (ERGEBNIS). (Quelle: http://www.materialsgate.de/mnews/10225/Abstract+SintercladdingEin+neues+Verfahren+für+verschleißbeständige+Beschichtungen.html, Stand 28.03.2012, Hervorhebungen durch IBL)

Das fachliche Problemfeld und die Untersuchungsverfahren werden unmittelbar in den beiden einleitenden Sätzen genannt. Satz 3 verweist auf das genutzte Verfahren, Satz 4 weist auf die qualitativen Vorteile des neuen Verfahrens hin.

Während ein informatives Abstract für Nichtfachleute auf Grund der unbekannten Fachlexik und der genutzten Prozesse meist unverständlich ist, haben Fachleute mit dem Text keine Verständnisprobleme, da die Kontextinformationen durch ihre Vorkenntnisse klar sind. Ihr Interesse gilt der Versuchsanordnung und dem Ausgang des Versuchs, um Schlussfolgerungen für eigene Studien ziehen zu können. Der interessierte Leser wird sich nach dem Lesen des Abstracts genauer mit dem vorgestellten technischen Prozess durch Lektüre des Fachbeitrags auseinander setzen. Der Fokus eines informativen Abstracts liegt folglich auf der Wiedergabe von Inhalten und nicht auf der Darstellung der Abfolge von Textpassagen wie dies bei einem indikativen Abstract der Fall ist.

In der fachkommunikativen Praxis sind je nach Fachgebiet, Untersuchungsgegenstand, Konvention und Abstraktionsgrad von Zeitschriften häufig Mischformen aus indikativem und informativem Abstract anzutreffen. "Das informativ-indikative Referat ist eine Mischform, die den Benutzer über ausgewählte Sachverhalte informiert und andere Sachverhalte nur erwähnt." (DIN-1426 1988: 3) Allerdings scheinen Abstracts in den Natur- und Technikwissenschaften auch aufgrund der von den Publikationsorganen standardisierten Autorenhinweise stärker informativ als indikativ $\mathrm{zu}$ sein, wohingegen Abstracts in den Geisteswissenschaften und auch Konferenzabstracts (als prospektive Texte) stärker indikativen Charakter tragen (vgl. Busch-Lauer 2001).

Im Gegensatz zum reinen Textabstract hat sich das Strukturreferat unter dem Einfluss der Dokumentationswissenschaften und renommierter angloamerikanischer Fachzeitschriften besonders in der Medizin etabliert. Die Vorgabe von standardisierten Textelementen erspart dem Autor die aufwändige Formulierung von Ziel, Gegenstand, Methode und Schlussfolgerungen. Die Autoren konzentrieren sich auf die wesentlichen Inhaltselemente eines Forschungsartikels. Dies ist nicht nur zeitökonomisch, sondern ermöglicht auch das schnelle Indexieren, das elektronische Erfassen von Daten in Forschungsdatenbanken und erleichtert zudem die Übersetzung des Textes durch den Autor bzw. professionelle Übersetzungsagenturen. 
Beispiel 3 aus der Medizin veranschaulicht diese Möglichkeit der Informationsstrukturierung und -kondensation über die Teilüberschriften: Hintergrund und Fragestellung, Methode, Ergebnisse, Schlussfolgerung. In jedem Abschnitt treten dafür typische Kollokationen auf, z. B. Das Thema ... wird diskutiert...; Neben den relativ intensiven Debatten... fehlt eine kritische Auseinandersetzung (THEMA und FORSCHUNGSLÜCKE).

Beispiel (3):

\begin{abstract}
Hintergrund und Fragestellung: Das Thema Rationierung im Gesundheitswesen wird zunehmend auch in der deutschen Ärzteschaft diskutiert. Neben den relativ intensiven Debatten über die Entscheidungsfindung auf der Makroebene des Gesundheitssystems fehlt eine kritische Auseinandersetzung darüber, wie Ärzte auf der Mikroebene in der individuellen Arzt-Patienten-Beziehung die unvermeidlichen und bereits heute prävalenten Rationierungen in einer medizinisch und ethisch vertretbaren Weise durchführen können. Die Entwicklung praxistauglicher Lösungsansätze für die Mikroebene bedarf im Vorfeld sozialempirischer Studienansätze, um ein differenzierteres Bild der Verbreitung und Häufigkeit ärztlicher Rationierung sowie ein tiefergehendes Verständnis der dabei zu erwartenden Probleme zu gewinnen.
\end{abstract}

Methode: Unter Verwendung des deutschen Krankenhausregisters wurde eine bundesweite Fragebogenstudie an einer Zufallsstichprobe von 1137 Klinikern in den Bereichen Intensivmedizin und Kardiologie durchgeführt.

Ergebnisse: Über drei Viertel der befragten Ärzte gaben an, Patienten nützliche Maßnahmen aus Kostengründen vorenthalten zu müssen. Allerdings berichtete nur ein geringer Anteil dieser Gruppe, dass es sich dabei um ein häufiges Phänomen handele. In Bezug auf Fachbereich, ärztlichen Status und Trägerschaft lassen sich z. T. signifikante Unterschiede in der Häufigkeit von Rationierungsentscheidungen feststellen. Die große Mehrheit der Kliniker beschrieb negative Auswirkungen der Mittelknappheit auf ihre Arbeitszufriedenheit und auf die ArztPatienten-Beziehung. Deutsche Kliniker sehen kaum noch Möglichkeiten für effizienteres Arbeiten als Alternative zu Rationierungen. Deutlich mehr als die Hälfte sah demgegenüber die Bereitstellung von zusätzlichen Mitteln für den Gesundheitssektor durch höhere Krankenkassenbeiträge oder einen höheren finanziellen Eigenanteil der Patienten als einen akzeptablen Weg im Umgang mit der Mittelknappheit.

Schlussfolgerung: Es bedarf der Weiterentwicklung von expliziten Instrumenten der Leistungsbegrenzung, welche unvermeidliche Rationierungsentscheidungen auf der Mikroebene ersetzen oder entlasten. Entsprechende Ansätze müssen ergänzt werden durch Beratungsstrukturen zur Unterstützung eines rationalen und moralisch akzeptablen ärztlichen Umganges mit Mittelknappheit. (Quelle: Deutsche Medizinische Wochenschrift 2009; 134(24): 1261-1266, Hervorhebungen durch IBL)

\title{
2.3 Anleitungen zum Verfassen von Abstracts
}

In den vergangenen Jahren haben sich durch die wachsende Informationsflut, den Konkurrenzdruck von Zeitschriften, den Publikationsdruck der Wissenschaftler und den Übergang von Print- zu Online-Medien auch Veränderungen in den Anleitungen für das Verfassen von Abstracts vollzogen. Vergleicht man Fachzeitschriften verschiedener Fachdisziplinen bzw. Abstraktionsniveaus mit Blick auf die dazu formulierten Richtlinien, zeigt sich, dass die Angaben und Präzisionsgrade für die Abfassung von Abstracts zwar noch differieren (vgl. Busch-Lauer 2001), aber auf eine stärkere Normierung innerhalb einer Wissenschaftsdisziplin hinauslaufen. So fordert die Deutsche Medizinische Wochenschrift in ihren Autorenrichtlinien durchgängig strukturierte Abstracts, (vgl. http://www.thieme.de/SID29EE8BAE-93890EA3/local_pdf/DMW/autorenrichtlinien.pdf, Stand 08.03.2012), wohingegen die Autorenrichtlinien für technische Fachzeitschriften textuelle Abstracts mit einer Limitierung in Bezug auf die Wort- bzw. Zeilenzahl (ca. 10-15 Zeilen oder ca. 100-150 Wörter) festlegen. In der Medizin sind die Hinweise sehr restriktiv. So folgt die renommierte 
Deutsche Medizinische Wochenschrift in ihrem Anforderungsprofil sehr stark den führenden englischsprachigen medizinischen Zeitschriften sowie den Empfehlungen der Ad hoc Working Group (1987) ${ }^{1}$. Im Vergleich dazu enthalten die meisten in Deutsch publizierten linguistischen Zeitschriften in den Hinweisen für Autoren relativ vage Formulierungen für die Gestaltung von Abstracts (vgl. Busch-Lauer 2001).

Betrachtet man jedoch im Vergleich dazu die Anforderungen an Abstracts für die Teilnahme an Fachtagungen, so sind diese seit Jahren in allen Fachdisziplinen sehr viel strenger formuliert, unterliegen einem Peer Review, und damit auch einer inhaltlichen und formalen Qualitätssicherung.

Insgesamt haben Studien $\mathrm{zu}$ den Arten und Richtlinien für Abstracts gezeigt, dass offensichtlich ein Zusammenhang zwischen der zu verarbeitenden Informationsmenge in einem Fach, der Notwendigkeit der Informationserfassung in Form von Abstracting Journals und dem Grad der Normierung von Textsorten in Form von Richtlinien besteht. Die Formulierung einheitlicher Richtlinien für Abstracts in akademischen Schreibkursen erscheint deshalb nicht sinnvoll, sondern muss in Abhängigkeit vom Zweck, dem Adressatenkreis und der Fachdisziplin erfolgen. Für Schreibnovizen empfehlen sich daher Modelltexte und zunächst orientierende Darstellungen, wie z.B. Philipp Koopman ("How to write an abstract"), vgl. http://www.ece.cmu.edu/ koopman/essays/abstract.html, Stand 08.03.2012).

\section{$3 \quad$ Abstracts aus linguistischer Sicht}

\subsection{Linguistische Untersuchungen zur Textsorte Abstract}

Aufgrund ihrer kommunikativen Relevanz unterliegt die Textsorte Abstract in der Praxis häufig der kritischen Betrachtung durch Fachleute und Dokumentationswissenschaftler. Auch die Linguistik hat sich verstärkt mit den sprachlichen, inhaltlichen und interkulturellen Unzulänglichkeiten auseinander gesetzt. Die folgende Tabelle gibt einen Überblick zu ausgewählten linguistischen Arbeiten und ihren Untersuchungsgegenständen:

Untersuchungsgegenstand

Überblicksdarstellungen

Kommunikative Funktion Makrostruktur

(Teiltextsegmente + Sprachmittel und Merkmale Einzelne Fachgebiete

Verschiedene Arten von Abstracts/ Konferenzabstracts/ Extended Abstract

Kulturspezifik und

Übersetzungswissenschaft

\author{
Autoren \\ Fluck (1988); Gnutzmann (1991); Ickler (1993); Jordan (1991); Ventola \\ (1994) \\ Fluck (1989); Gibson (1992); Golebiowski (2009); Dahl (2009) \\ A. Oldenburg (1991) Maschinenbau, Pädagogik, Baßler (2000) Soziologie, \\ Gnutzmann (1991) Passiv; Webber (1996) Metaphern, verschiedene \\ Fachgebiete, Medizin; Dayrell (2009) Verben \\ Ruge (1992) Physik, Informatik und Chemie, Salager-Meyer (1990) Medizin, \\ Santos (1996) Angewandte Linguistik, Tibbo (1992) Chemie, Psychologie, \\ Geschichte, Baßler (2000) Soziologie, Busch-Lauer 2001 Medizin, \\ Linguistik \\ Adolphi (1996), Geowissenschaften, Tsai (2010) Abstracts von Patenten
}

Ventola (1994); Andersson/Gunnarson (1995); Kourilová (1996), Melander (1997); Melander/ Swales/Fredrickson 1994), Baßler (2000), Busch-Lauer (2001); Martín-Martín (2005); Lorés-Sanz (2009); Müller (2008); Tsai (2009)

\footnotetext{
1 Die Ad hoc Working Group (in den USA gegründete, internationale Arbeitsgruppe aus Medizinern, Dokumentationsmitarbeitern und Verlagsrepräsentanten) hat zur Effektivierung von Dokumentation und Publikation in der Medizin für die in der Medizin am häufigsten verwendeten Textsorten (Originalartikel, Fallstudie - Kasuistik, Übersichtsartikel) Empfehlungen erarbeitet, die von den meisten internationalen Verlagen als Standard für Strukturabstracts übernommen wurden. Abstracts zu Experimentalstudien/ Originalartikeln folgen demnach dem Muster: Objective (Ziel), Design (Forschungsdesign), Setting (Duchführung), Patients (Patienten), Interventions (Medikation und Behandlungsverlauf), Main outcome measures (Ergebnisübersicht), Results (Ergebnisse im Detail), Conclusions (Schlussfolgerungen).
} 
$\begin{array}{ll}\text { Fremdreferate } & \text { Endres-Niggemeyer/Hobbs/Sparck (1995) } \\ \text { Verfassen von Abstracts } & \text { Cargill/O'Cpnnor (2009); Feak/Swales (2009), Skern (2009) }\end{array}$

Tabelle 1: Linguistische Untersuchungen zur Textsorte Abstract

Als problematisch für die Produktion von Abstracts hat sich in der Praxis einerseits die Informationskomprimierung erwiesen und die Schwierigkeit, die notwendige Information von redundanten Informationselementen $\mathrm{zu}$ trennen und andererseits eine optimale Vertextung durchzuführen. Während diese Prozesse für die Muttersprache aufgrund der jeweiligen Schreibsozialisation nicht so schwierig erscheinen, ist dieser Prozess in der Fremdsprache Englisch offenbar erschwert (Ventola 1994, Kourilová 1996, Busch-Lauer 2001). Die Gründe dafür sind noch nicht ausreichend erforscht. Es ist jedoch $\mathrm{zu}$ vermuten, dass die Wissenschaftstraditionen eines Landes, die damit verbundenen tradierten Schreibkonventionen und Unterschiede im Sprachsystem wichtige Indikatoren für Schreibprobleme sind (vgl. Busch-Lauer 2001). Kourilová (1996: 16f.) hat linguistische und interkulturelle Schreibprobleme von Nichtmuttersprachlern (NNS) im Englischen untersucht und bemerkt dazu:

Abstracts written by NNS appear to be disorganised, incohesive, out of focus because the rhetoric and/or thought processing violate the NS's expectations of linear development. Lack of proficiency in handling the development of the author's argument, digressions, lack of focus and specification of findings and ideas, deficient logic resulting in a poor flow of ideas present the greatest potential for abstracts written by Slovak authors to be dismissed as incoherent and unprocessable. (Kourilová 1996: 16f.)

Außerdem nennt sie z. B. auch psychologische Aspekte wie 'overconfidence' (große Selbstsicherheit/Missachtung anderssprachiger Regularitäten); 'overcaution' (Unschlüssigkeit/Vagheit in der Darstellung); status seeking (Wettbewerbsdruck/ Statussymbole), die zur Beeinträchtigung der Lesbarkeit von Abstracts und damit auch allgemein zur Rezeption von Fachartikeln in der Fremdsprache führen können.

Sicher stellen die oben genannten Faktoren ein generelles Problem beim Schreiben in der Fremdsprache dar. Sie offenbaren sich jedoch stärker in Abstracts als in anderen Wissenschaftstexten, da diese Texte in Form von Konferenzabstracts oder Inhaltsangaben häufig durch Gremien ohne die Vorlage/Hinzuziehung des Primärtextes evaluiert werden und ihre Qualität ausschlaggebend ist für eine Publikation oder die Annahme eines Tagungsbeitrags. Das heißt, die Reputation eines Wissenschaftlers oder Forscherteams kann durch die Nichtakzeptanz von Abstracts als Folge der oben beschriebenen Mängel durchaus in Mitleidenschaft gezogen werden. Zur Vorbeugung solcher Probleme sollten z. B. Nichtmuttersprachler des Englischen die jeweils vorgegebenen sprachlich-textuellen Anforderungen besonders für die von ihnen in L2 (Englisch) formulierten Texte beachten und diese Texte einer besonders sorgfältigen Textrevision (z. B. auch durch einen Muttersprachler) unterziehen. Zusätzlich können auch die folgenden nach ISO und DINNorm 1426 (1988: 2) definierten fünf Kriterien als Qualitätsmerkmale herangezogen werden:

- Vollständigkeit (alle wesentlichen Aspekte eines Dokumentes sind ohne Rückgriff auf das Original für den Fachmann verständlich)

- Genauigkeit (Inhalt +Aussage des Abstracts sollten mit dem Original übereinstimmen)

- Objektivität (Abstracts sollten wertneutral sein)

- Kürze (Abstracts sollten so kurz wie möglich sein; Länge ist auf 200-250 Wörter zu beschränken) 
- Verständlichkeit (z. B. Verwendung national und international eingeführter Nomenklaturen etc.).

Betrachten wir im folgenden Abschnitt, wie sich die beschriebenen Kriterien in den bislang vorliegenden Untersuchungen und in der Struktur eines Beispieltextes widerspiegeln.

\subsection{Länge von Abstracts}

In der Regel sollten Abstracts nach den Hinweisen für Autoren in Fachzeitschriften ca. 200250 Wörter für Originalarbeiten und Übersichtsartikel nicht überschreiten. Abstracts für Konferenzbeiträge sind in der Regel auch auf 200 Wörter begrenzt.

Melander (1997) hat in seiner Untersuchung zu muttersprachigen englischen (L1) und fremdsprachigen englischen Abstracts (L2) sowie zu schwedischen L1-Abstracts aus drei Fachgebieten den Durchschnittswert von 155 Wörtern ermittelt (Biologie 164, Medizin 153 und Linguistik 149) Wörter. In seinem Korpus sind die Abstracts amerikanischer Autoren (L1) insgesamt deutlich länger als die schwedischer Abstracts (L1). Die L2-englischen Abstracts schwedischer Autoren sind in den biologischen und medizinischen Abstracts kürzer als die L1-Texte, in der Linguistik sind sie sogar am kürzesten. Melander verweist als Ursache für diese Unterschiede auf den unterschiedlichen Charakter der Zeitschriftenartikel (Experimentalstudie versus Erörterungen) und auf die Vorgaben der Zeitschriftenverlage sowie die Form der Abstracts (Strukturabstract versus Textabstract).

Tibbo (1992: 41) ermittelte bei ihrem Vergleich englischer L1-Abstracts in verschiedenen Fachgebieten die folgenden durchschnittlichen Textlängen: Chemie 137 Wörter, Psychologie 141 Wörter, Geschichtswissenschaft 80 Wörter.

Für das von mir verwendete Materialkorpus aus medizinischen, linguistischen und technischen Abstracts (vgl. Busch-Lauer 2001 und 2007) konnte ich eine durchschnittliche Länge von ca. 155 Wörtern ermitteln. Die medizinischen Abstracts waren auch als Strukturreferat oft länger als die linguistischen, die technischen Abstracts waren in der Regel am kürzesten. Dies ist auf den Untersuchungsgegenstand, die Methode, aber auch auf Manuskriptvorschriften und in der Technik auf formelhafte Ausdrucksmöglichkeiten zurückzuführen. Darüber hinaus dominieren in der Linguistik und Technik Textabstracts, in der Medizin jedoch Strukturabstracts.

\subsection{Teiltextsegmente und Textbausteine in Abstracts}

Vollständigkeit, Genauigkeit und Objektivität eines Abstracts lassen sich durch Fachleute natürlich besser einschätzen als durch Linguisten. Dennoch ist es möglich, über die Analyse des Aufbaus von Abstracts auch Aussagen zu diesen Qualitätskriterien und zur Verständlichkeit zu treffen.

Tibbo (1992) hat die als "coding categories" bezeichneten Teiltextelemente (1) Background, (2) Purpose/Scope, (3) Hypotheses, (4) Methodology, (5) Results, (6) Conclusions und (7) Others in englischen Abstracts verschiedener Fachgebiete untersucht. Für die Abstracts aus der Chemie konnte sie die Häufigkeitsabfolge 4-2-5-6-1, für die Psychologie die Abfolge 5-46-2-7-3-1 und für die Geschichtswissenschaft 7-6-2-4-1-5-3 ermitteln. Dies deutet darauf hin, dass bei der Gestaltung von Abstracts zwischen den Disziplinen unterschiedliche fachliche Maßstäbe bestehen. Während in der Chemie die "Methodologie", das "Ziel der Untersuchung" sowie die "Ergebnisse" im Vordergrund stehen, sind in der Psychologie zunächst die "Ergebnisse" (43\%-Anteil) und dann die "Methodologie" sowie die Schlussfolgerungen von Interesse. Noch größere Diskrepanzen zeigen sich in der Geschichtswissenschaft, wo 64\% der Sätze sich keiner der Kategorien zuordnen lassen und die "Schlussfolgerungen" mit 14\% Anteil die vom Darstellungsumfang zweitgrößte Kategorie vor den "Ergebnissen" bilden. 
Hutz (1997) hat in seiner Untersuchung zu Abstracts in der Sozialpsychologie die folgenden Teiltexte festgestellt: (1) Einführung in das Forschungsgebiet und Zielsetzung der Studie; (2) Angaben zu experimentellen und methodischen Grundlagen; (3) Darlegung der wichtigsten Untersuchungsergebnisse und (4) Diskussion der Ergebnisse. Für sein Korpus konnte er die Häufigkeitsabfolge 3-2-1-4 ermitteln.

Melander (1997) verweist in seiner Untersuchung zu den Fachgebieten Biologie, Medizin und Linguistik darauf, dass die biologischen Abstracts in seinem Korpus dem Muster "MethodeErgebnisse" folgen, die medizinischen und linguistischen sich jedoch in den Sprachen unterscheiden: schwedische medizinische Abstracts folgen dem Muster "ProblemstellungEmpfehlung", die L2-englische Abstracts dem Muster "Methode-ErgebnisseSchlussfolgerungen" und die amerikanisch-englischen Abstracts waren länger und als Strukturreferate mit der Abfolge (Ziel-Methode-Ergebnisse-Schlussfolgerungen) formuliert. Für die linguistischen Abstracts konnte Melander (1997) die größte Variationsbreite zwischen den Korpora nachweisen. Auch Santos (1996) hat für die 96 untersuchten linguistischen Abstracts sehr variable Anordnungen von Teiltexten aufgezeigt. Diese Ergebnisse deuten darauf hin, dass die Vertextung in den Naturwissenschaften und in der Medizin offenbar einer stärkeren Normierung unterliegt als in den Sozial- und Geisteswissenschaften (vgl. dazu auch Martín-Martín 2005).

H. Oldenburg (1992) und A. Oldenburg (1995) entwickelten (ähnlich dem Modell der "moves" and "steps" von Swales 1990) das Konzept der Teiltextsegmente (TTS). Nach A. Oldenburg (1995: 111) sind TTS "relativ autonome inhaltlich-funktionale Einheiten unterhalb der Teiltextebene, deren Beginn bzw. Ende [...] durch Gliederungssignale angezeigt werden kann". Sie treten in bestimmten Anordnungen innerhalb des Teiltextes auf und so ist es möglich, auf der Grundlage von Textanalysen an größeren Textkorpora zu TTS-Typen zu gelangen, die sprach- und fächerübergreifend gleichartig oder verschieden sein können (vgl. Tabelle 2).

Autor

A. Oldenburg (1991: 70-75):

Hutz, (1997: 107f.)

Sozialpsychologie

Deutsch

Englisch

$\begin{array}{ll}\text { Ad-Hoc Working } & \text { Medizin } \\ \text { Group (1987) } & \text { Englisch }\end{array}$

\section{Teiltextsegmente}

(1) Globale Charakterisierung des Forschungsfeldes und/oder der Forschungssituation

(2) Hauptziel/Hauptuntersuchungsgegenstand der Arbeit

(3) Darstellung der Untersuchungsergebnisse

(4) Methoden/Modelle/Experimente/ Verfahrensschritte

(5) Konsequenzen für die Forschung und Praxis

(1) Einführung in das Forschungsgebiet und Zielsetzung der Studie;

(2) Angaben zu experimentellen und methodischen Grundlagen;

(3) Darlegung der wichtigsten Untersuchungsergebnisse

(4) Diskussion der Ergebnisse.

Original article: Objective, Design, Setting, Patients or Participants, Interventions, Main Outcome Measures, Results \& Conclusion

Tabelle 2: Übersicht zu den Teiltextsegmenten in verschiedenen Fachgebieten 
Wie aus Tabelle 2 ersichtlich ist, folgen Abstracts, die auf Experimenten und Versuchen beruhen, in ihrem Textaufbau der Anordnung und Durchführung des Experiments: (1) Aufzeigen der Forschungslücke; (2) Zielstellung, (3) Material und Methodik der Untersuchung und (4) Ergebnisse und Schlussfolgerungen.

Die Analyse der TTS in meinem Korpus aus linguistischen, medizinischen und technischen Texten wurde in folgenden Schritten durchgeführt: (1) Markierung der Teiltexte und Textbausteine in den einzelnen Abstracts; (2) Bestimmung der Funktion der Strukturen im Text; (3) Abstraktion der Funktionen und Ermittlung der sprachlichen Ausdrucksmöglichkeiten.

Betrachten wir dieses Verfahren anhand eines deutschsprachigen Beispieltextes aus der Technik (vgl. Busch-Lauer 2007: 111). Die Teiltextfunktionen werden durch Fettdruck in Klammern angegeben.

\section{Beispiel (4):}

Bekanntermaßen führen Unterschiede in der Partikelgrößenverteilung von Pigmenten zu Unterschieden in der Teilchenpackung und in der Mikrostruktur von Papierstrichen. (EINORDNUNG IN DAS FORSCHUNGSFELD) Es wurde bereits eine Vielzahl von Untersuchungen durchgeführt, um den Zusammenhang zwischen Strichstruktur und Bedruckbarkeitseigenschaften $\mathrm{zu}$ ermitteln. Allerdings konzentrierte sich das Interesse überwiegend auf Modellpigmente oder relativ isotrope Pigmente, wie rhomboedrische PCCTypen, während Arbeiten zur systematischen Bewertung von Kaolinen nur in minimalem Umfang vorliegen. (AUFZEIGEN DER FORSCHUNGSLÜCKE) Bei der vorliegenden Untersuchung wurden an Kaolinstrichen die Veränderungen in der Strichstruktur während des Glättvorgangs systematisch erforscht. (REFERENZ AUF DIE ZIELE UND METHODEN DER EIGENEN UNTERSUCHUNG) Die Untersuchungen bezogen sich auch auf Veränderungen in der Oberflächenrauhigkeit, Partikelorientierung und Porenstruktur des Strichs und diese Veränderungen wurden zum Farbwegschlag und zur Druckglanzentwicklung in Beziehung gesetzt. Anschließend wurde bei einer Reihe von maßgeschneiderten Kaolinen die Beziehung zwischen Porenstruktur und Bedruckbarkeit untersucht. (VERWENDETES UNTERSUCHUNGSVERFAHREN) Die Ergebnisse bestätigen die Bedeutung des Pigmentdesigns für eine optimale Bedruckbarkeit des Strichs. (KURZDARSTELLUNG DES ERGEBNISSES UND SCHLUSSFOLGERUNG) (Quelle: Druck und Druckindustrie, 2/2000, zit. nach Busch-Lauer 2007: 111, Hervorhebungen durch IBL)

Durch Abstraktion kann man aus einem Textkorpus die für ein Fachgebiet typische Anordnung von Teiltextsegmenten ermitteln. Exemplarisch wird dies für unser Beispiel in Tabelle 3 mit Erfassung der deutschen und englischen Sprachmittel (Textbausteine) dargestellt.

$\begin{array}{llll}\text { TTS } & \text { TTS-Komponente } & \text { Deutscher Text } & \text { Englischer Text } \\ 1 & \begin{array}{l}\text { Einführung in das For- } \\ \text { schungsgebiet/Zielsetzung }\end{array} & \begin{array}{l}\text { Bekanntermaßen führen } \\ \text { Unterschiede in der ... zu } \\ \text { Unterschieden in ... }\end{array} & \begin{array}{l}\text { It is well known that differences in ... } \\ \text { lead to ... and ... }\end{array} \\ & \begin{array}{l}\text { Bezug auf vorliegende Unter- } \\ \text { suchungen }\end{array} & \begin{array}{l}\text { Es wurde bereits eine } \\ \text { Vielzahl von Untersu- } \\ \text { chungen durchgeführt, um } \\ \text { den Zusammenhang } \\ \text { zwischen ... und ... zu } \\ \text { ermitteln. }\end{array} & \begin{array}{l}\text { Many studies have also been carried } \\ \text { out to investigate the link between ... }\end{array} \\ & \end{array}$




\begin{abstract}
Mängel vorliegender Untersuchungen
\end{abstract}

2

\section{Darlegung der wichtigsten Untersuchungsergebnisse}

Allerdings konzentrierte sich das Interesse überwiegend auf ... oder..., (wie ...), während Arbeiten zur systematischen Bewertung von ... nur in minimalem Umfang vorliegen.

Bei der vorliegenden Untersuchung wurden an ... die Veränderungen ... während des ... systematisch erforscht.

Die Untersuchungen bezogen sich auch auf Veränderungen in der ... und diese Veränderungen wurden zum ... und zur ... in Beziehung gesetzt.
However, most of these studies have concentrated on ... or ... (such as ...) and relatively little work has been carried out to assess ... in a systematic way.

In this study, the systematic changes in ... were investigated for ...

Variations in the ... were assessed, and these changes related to the ... and ...

The relationship between ... and ... was then investigated for a range of

Anschließend wurde bei einer Reihe von maßgeschneiderten ... die Beziehung zwischen ... und ... untersucht

Die Ergebnisse bestätigen The results illustrate the importance die Bedeutung des ... für ... of ... if ... is to be obtained.
Diskussion der Ergebnisse bzw. Hauptschlussfolgerung

Tabelle 3: Teiltextsegmente und Textbausteine im Beispielabstract (4)

Vergleicht man die Anordnung und den Umfang der TTS in Abstracts verschiedener Fachgebiete und Sprachen miteinander, zeigen sich durchaus Parallelitäten und Unterschiede (vgl. Busch-Lauer 2001; Martín-Martín 2005), die darauf hindeuten, dass es offenbar unterschiedliche Schreibsozialisationen gibt.

\subsection{Zur Vermittlung der Textsorte Abstract im fachbezogenen Fremdsprachenunter- richt}

Die Ergebnisse der in den Kapiteln 3.2-3.3 beschriebenen linguistischen Untersuchungen können für Fachleute, die sich mit Abstracts unter Produktions- und Textqualitätsgesichtspunkten auseinandersetzen müssen, aber auch für Studierende interessant sein. Die Kenntnis der Textsortenspezifika ist zum Beispiel für Studierende der Fakultät Physikalische Technik an der Westsächsischen Hochschule in Zwickau Voraussetzung, um kommunikationsadäquat deutsch- und englischsprachige Abstracts zu ihren Bachelorabschlussarbeiten zu verfassen. Aufgrund der gedrängten Modulstruktur der Studiengänge bietet es sich in der Ausbildungspraxis an, die Textsorte und das Verfassen von Abstracts in die fachbezogene Fremdsprachenausbildung Englisch zu integrieren. Das seit ca. 2 Jahren praktizierte Verfahren (in Anlehnung an die Darstellung von Busch-Lauer 2004: 335f.) hat sich bewährt. Es sind im Vergleich zu den Vorjahren qualitativ hochwertige Texte entstanden und die Bewusstheit der Studierenden für fremdsprachige Schreibprozesse hat sich signifikant verbessert. Im Folgenden soll das eingesetzte Verfahren kurz skizziert und die Qualität der entstandenen Abstracts an zwei Beispielen aufgezeigt werden.

Im Rahmen der fachbezogenen Englischausbildung ist als mündlicher Prüfungsteil (wie in einer realen Konferenzsituation) pro Student ein Fachvortrag im Umfang von 15 Minuten zu halten und dazu vorab eine Textzusammenfassung (ein Abstract - im Umfang von ca. 200- 
250 Wörtern) an den Dozenten einzureichen. Das Abstract wird (nach ggf. erfolgter Korrektur durch den Dozenten) nach dem Vortrag in Kopie an alle Studierenden im Kurs ausgeteilt und in seiner Qualität bewertet. Dazu werden die beschriebenen Qualitätskriterien verwendet. Auf diese Weise gelingt es, die Studierenden für Textproduktionsprozesse und die Textqualität zu sensibilisieren.

In Vorbereitung auf den mündlichen Prüfungsteil werden die Studierenden im Seminar anhand von authentischen Modellabstracts von im Unterricht zu behandelnden Themen aus ihrem speziellen Fachgebiet (z. B. Mikrotechnologie, Energietechnik, Biomedizintechnik) vertraut gemacht. Über die Rezeption dieser Texte hinaus wird die Textsorte bestimmt und darauffolgend ihr textueller und inhaltlicher Aufbau. Durch die verschiedenen Modelltexte (indikative, informative und Strukturabstracts) erhalten die Studierenden eine Übersicht über die Vielfalt der Textausprägung dieser Textsorte. Durch die Bestimmung der Teiltextsegmente und der sprachlichen Indikatoren werden die Studierenden mit Formulierungsmustern vertraut (Kollokationen, Verbstrukturen etc.) und durch die Selektion der Terminologie auch mit dem Fachwortschatz. Die parallele Betrachtung von deutschen Ausgangstexten und englischen Übersetzungen offenbart außerdem Problemfelder von "wortwörtlichen" Übersetzungen. Ziel ist es, dass die Studierenden verstehen lernen, dass es auch als Schreibanfänger sinnvoller ist, fachbezogene Texte bei Bedarf gleich in der Fremdsprache Englisch zu verfassen, d. h. auf negativen Transfer beruhende Übersetzungsfehler zu vermeiden.

Für die Textproduktionsphase wird den Studierenden dann empfohlen, die folgenden Arbeitsschritte zu absolvieren:

\section{Schritt 1: Definition der Kommunikationssituation}

Zunächst sind der Adressatenkreis des Abstracts, der Zweck und der Umfang der Inhaltsreduktion zu bestimmen.

\section{Schritt 2: Analyse der Kerninformation des Vortrags und der Kernstruktur}

Anhand des Vortragsthemas wird entschieden, welcher Abstracttyp (ein indikatives, ein informatives oder ein Strukturabstract) am besten für die Darstellung geeignet ist. Dann werden die Kernaussagen des Vortrags möglichst in ihrer chronologischen Abfolge in der Präsentation erfasst. Bei Darstellung eines fachlichen Sachverhaltes fließen z. B. das Ziel des Beitrags, die Definition/fachliche Einordnung des Gegenstands, mögliche Entwicklungen und das Ergebnis/das zu lösende Problem in das Abstract ein. Bei Darstellung von Prozessen werden die Ziele, Abläufe und Ergebnisse sowie Vor- und Nachteile erfasst. Bei der Darstellung von Experimenten folgen die Studierenden der ihnen bereits vertrauten IMRAD-Struktur von Fachartikeln. Hilfreich sind in diesem Kontext z. B. Überschriften und Teilüberschriften sowie Kernsätze eines Abschnittes, so dass die gedankliche Struktur/Textstruktur des Abstracts festgelegt werden kann.

\section{Schritt 3: Text erstellen - Inhaltskomprimierung/-synthese}

Nach der Ermittlung, Auswahl und Entnahme von benutzerdefinierten Daten/Inhalten, werden Sätze gebildet, die nicht isoliert voneinander, sondern in Relationen stehen. Kohäsive und kohärenzstiftende Mittel sind so einzusetzen, dass ein in sich geschlossener Kurztext entsteht. Während des Abstraktionsprozesses sollten die Studierenden sorgsam redundante (überflüssige) von notwendigen Informationen trennen.

\section{Schritt 4: Textentwurf überarbeiten - Informationskontrolle}

Nachdem der Entwurf für das Abstract vorbereitet ist, sollen die Studierenden eine Textrevision durchführen, die sprachliche und kommunikative Aspekte einschließt. In diesem Kontext werden die Qualitätskriterien (Vollständigkeit, Genauigkeit, Objektivität, "Kürze" und Verständlichkeit) herangezogen. Gegebenenfalls lohnt es sich auch den entstandenen Text durch 
einen Kommilitonen gegenlesen zu lassen, so dass noch einmal eine Prüfung auf inhaltliche, formal-strukturelle und kommunikativ-funktionale Adäquatheit erfolgt und auch das Kriterium Verständlichkeit getestet wird.

Betrachten wir nun zwei Abstracttexte von Studierenden aus dem Bereich Mikrotechnologie, für die das oben beschriebene Verfahren genutzt wurde. Beide Abstracts können als informative Abstracts eingestuft werden. Beispiel 5 thematisiert Organische Leuchtdioden (OLEDs), ein Leuchtmittel mit Zukunft. Die wichtigen Elemente des Vortrags (Definition, Funktionsweise, Arten, Anwendung, Nutzen und Problemfelder) geben die Inhaltskategorien des Vortrags gelungen wieder.

Beispiel (5), Darstellung eines Sachgegenstandes:

\section{Organic Light-Emitting Diodes}

An OLED is an LED in which the emissive electroluminiscent layer is a film of organic compounds which emits light in response to an electric current. (DEFINTION/BENENNUNG $D E S$ GEGENSTANDES). This layer material is situated between two electrodes. One of these electrodes is tranparent.

An OLED display functions without a backlight. It can display deep black levels and can also be thinner and lighter than established liquid crystal displays. There are two main families of OLEDs: (1) those based upon small molecules and (2) those employing polymers. OLED displays can either use passive-matrix (PMOLED) or active-matrix addressing schemes. Activematrix OLEDs (AMOLED) require a thin-film transistor backplane to switch each individual pixel on or off, and can make higher resolution and larger size displays possible. (BESCHREIBUNG DER FUNKTIONSWEISE UND KLASSIFIKATION).

OLEDs are used in television screens, computer monitors, small, portable system screens such as mobile phones and PDAs, watches etc. OLEDs are also used in light sources for general space illumination and in large-area light-emitting elements. (ANWENDUNG IN DER PRAXIS)

In the future, OLEDs could replace LCDs and plasma screens in many applications. Their life-time is still a problem, because the red, green and blue dots of each pixel age at different rates. Due to this irregular wear and tear of the individual colours, there may develop colour shifts in the displays. Moreover, large screens are not available yet at a competitive price. So the breakthrough in television und monitor industries will still have to wait for several years to come. Problems also include the encapsulation of the components and the complex control of the pixel. (ZUKUNFT/PROBLEMFELDER)

In Textbeispiel 6 geht es um Recycling-Prozesse von Wafern. Es wird ein in Entwicklung befindlicher Prozess mit seinen Vor- und Nachteilen vorgestellt. Die Argumentation ist durch den Einsatz von Konjunktionen logisch und übersichtlich. Nach der Einordnung des Themas erfolgt die Problemdarstellung, die Prozessbeschreibung und die Abwägung der Vor- und Nachteile. Es fehlt ein Lösungsausblick, der aber aufgrund des Technikstandes auch nicht geliefert werden kann.

Textbeispiel (6), Darstellung eines Prozesses:

\section{Wafer Recycling in Semiconductor Fabrication}

Nowadays, recycling has a growing priority due to environmental protection and resource shortage. (RELEVANZ DES THEMAS)

In semiconductor fabrication, silicon wafers are used for production, electrical testing and quality assurance. Wafers are relatively expensive and it would not be economical to buy new wafers just for testing purposes. Therefore, the test wafers need to be recycled. (PROBLEM/L $\ddot{U C K E})$

There are two techniques: reclaim and wet chemical recycling. During the reclaim process, the surface of the wafer is ground off completely. Thus, all layers and even scratches can be 
eliminated. However, the wafer is thinned. In contrast, during wet chemical recycling, only the surface is etched. For each layer a different etchant is needed. Since you can process many wafers simultaneously, it is a very inexpensive process. (PROZESS-BESCHREIBUNG)

But what can you do against doping? There is intentional doping for the targeted modification of the electrical properties, but also unwanted, as the diffusion of copper. Currently, doping is not removable. One exception is copper. Copper oxide is formed on the surface and only this is etched. (PROBLEMBESCHREIBING IM PROZESS)

A benefit of the reclaim is that all layers and scratches can be removed. The drawback is that the wafer is getting thinner every time and thus its reuse is limited. A benefit of the wet chemical recycling, however, is that it is a very inexpensive process. The drawback is that a different etchant is required for each layer. (VOR- UND NACHTEILSZUSAMMENFASSUNG)

Vergleicht man den Aufbau der beiden Abstracts mit den von Fachleuten in Fachzeitschriften veröffentlichten Texten und ihrer Struktur, so zeigen sich aufgrund der Themenvielfalt zwar deutliche Unterschiede, aber es wird der hohe Qualitätsanspruch der Studierenden an ihre Texte deutlich. Die frühzeitige Sensibilisierung der Studierenden für die Textsorte bereitet sie gut auf eigenständige wissenschaftliche Publikationen vor. Nach ihrer eigenen Einschätzung waren die Instruktionen zur Textsorte und die durchgeführten Übungen zur Rezeption, Strukturierung und Produktion auch sehr nützlich für die Erarbeitung der Textzusammenfassungen für die Bachelorarbeit.

\section{$4 \quad$ Fazit}

Ziel des vorliegenden Beitrags war es, die Textsorte Abstract vorzustellen, die Typen von Abstracts aufzuzeigen und ihre textuellen und sprachlichen Merkmale zu beschreiben. Beispiele aus der Linguistik, Medizin und Technik haben strukturelle und interfachliche Unterschiede bei der Realisierung der Textsorte Abstract verdeutlicht. Darüber hinaus konnte anhand der Ergebnisse einiger linguistischer Arbeiten und an einer Beispielanalyse demonstriert werden, dass sich in der Textsorte Abstract wie auch in anderen Wissenschaftstexten unterschiedliche fachbezogene und idiosynkratische Schreibstile zeigen.

Das in der fachbezogenen Fremdsprachenausbildung verwendete Übungsprogramm zur Vermittlung der Textsorte Abstract hat sich bewährt, die Sensibilisierung der Studierenden für die Qualitätskriterien eines Textes wurde geweckt und die Fähigkeit der Studierenden zur Formulierung von Texten in der Fremdsprache geschult.

Weiterführende Untersuchungen zur Textsorte Abstract sollten insbesondere den medialen Einfluss der Online-Publikation von Abstracts (z. B. unter Einbeziehung von Verlinkungen) betrachten und die Funktion von Peer Review-Prozessen für die Gestaltung von Konferenzabstracts in der Mutter- bzw. auch in der Fremdsprache Englisch.

\section{Literatur}

Ad hoc working group for the critical appraisal of the medical literature (1987): "A Proposal for more informative abstracts of clinical articles." Annals of Internal Medicine 106: 598604.

ANSI/ISO Standard = American National Standard Institute (1979): "American national standard for writing abstracts". Tech. Rep. No.Z39, 14-1979. New York: ANSI 1987.

Adolphi, Katrin (1996): "Eine fachliche Textsorte in ihren Bezügen und Abgrenzungen: Die Textsortenvariante >Extended Abstract $<"$. In: Kalverkämper, Hartwig/Baumann, KlausDieter (eds.): Fachliche Textsorten. Komponenten - Relationen - Strategien. Tübingen: Narr: 478-500.

Andersson, Bo/Gunnarsson, Britt-Louise (1995): "A contrastive study of text patterns in conference abstracts". In: Wårwik, Brita/Tanskanen, Sanna-Kaisa/Hiltunen, Risto (eds.): 
Organization in Discourse. Proceedings from the Turku Conference. University of Turku: 139-148.

Auer, Peter/Baßler, Harald (eds.) (2007): Reden und Schreiben in der Wissenschaft. Frankfurt a. M./New York: Campus-Verlag.

Baßler, Harald (2000): "Wissenschaftsdiskurs im Vergleich: deutsche und russische Zeitschriftenabstracts der Soziologie." Vortrag zur LSF in Freiburg, http://fips.igl.uni-freiburg.de/auer/mod.php?mod=userpage\&page_id=36, Stand 17.1.2005.

Baßler, Harald (2003): "Russische, deutsche und angloamerikanische Zeitschriftenabstracts der Soziologie: Worin unterscheiden sie sich?" In: Gruber, Helmut/Menz, Florian/Panagl, Oswald (eds.): Sprache und politischer Wandel. Frankfurt a. M. et al., Lang: 189-212.

Berkenkotter, C./Huckin, Thomas (1995): Genre knowledge in disciplinary communication. Cognition/Culture/Power. Hillsdale, NJ: Lawrence Erlbaum.

Busch-Lauer, Ines-Andrea (2001): Fachtexte im Kontrast. Eine linguistische Analyse zu den Kommunikationsbereichen Medizin und Linguistik. Frankfurt a. M. et al.: Lang. (= Leipziger Fachsprachen-Studien 14).

Busch-Lauer, Ines-Andrea (2004): "Textbausteine in Abstracts." In: Wolff, Armin/Ostermann, Torsten/Chlosta, Christoph (eds.): Integration durch Sprache. Regensburg: FaDaF: 329-347. (= Materialien Deutsch als Fremdsprache 73).

Busch-Lauer, Ines-Andrea (2007): "Abstracts." In: Auer, Peter/Baßler, Harald (eds.): Reden und Schreiben in der Wissenschaft. Frankfurt/New York, Campus-Verlag: 99-114.

Cargill, Margaret/O'Connor, Patrick (2009): Writing Scientific Research Articles: Strategy and Steps. Chichester: Wiley-Blackwell.

Dahl, Trine (2009): "Author Identity in Economics and Linguistics Abstracts." In: SuomelaSalmi, Eija/Dervin, Fred (eds.): Cross-Linguistic and Cross-Cultural Perspectives on Academic Discourse. Amsterdam/Philadelphia Benjamins: 123-134. (= Pragmatics \& Beyond New Series 193).

Dayrell, Carmen (2009): "Sense-Related Verbs in English Scientific Abstracts: A CorpusBased Study of Students' Writing." ESP across Cultures 6: 61-78.

DIN 1426 (1988): Inhaltsangaben von Dokumenten. Kurzreferate, Literaturberichte. Berlin: Deutsches Institut für Normung.

DMW (1996): Deutsche Medizinische Wochenschrift. Manuskriptvorschriften für Autoren. Berlin. http://www.thieme.de/dmw/inhalt/service/autrili/autrili.htm, Stand 19.1.2011.

Endres-Niggemeyer, Brigitte et al. (1995): Summarizing text for intelligent communication. Dagstuhl. (= Dagstuhl-Seminar-Report 79).

Feak, Christine/Swales, John M. (2009): Abstracts and the Writing of Abstracts. Michigan: University of Michigan Press. (= Michigan Series in English for Academic \& Professional Purposes).

Fluck, Hans-Rüdiger (1988): "Zur Analyse und Vermittlung der Textsorte Abstract". In: Gnutzmann, Claus (ed.): Fachbezogener Fremdsprachenunterricht. Tübingen, Narr: 6790. (= Forum für Fachsprachenforschung 6).

Fluck, Hans-Rüdiger (1989): "Vergleichende Analyse deutschsprachiger Abstracts in wissenschaftlichen Zeitschriften". In: Laurén, Christer/Nordman, Marianne (eds.): Special languages, from human thinking to thinking machines. Clevedon, Multilingual Matters, 291-307.

Gallagher, John D. et al. (2008): Writing in English: A Guide for Advanced Learners. Stuttgart: UTB.

Gibson, T. R. (1992): Towards a discourse theory of abstracts and abstracting. Ph.D. thesis, Nottingham.

Gläser, Rosemarie (1990): Fachtextsorten im Englischen. Tübingen: Narr. (= Forum für Fachsprachenforschung 13). 
Gläser, Rosemarie (1991): "The LSP genre abstract revisited". ALSED LSP Newsletter 13, June: $3-10$.

Gnutzmann, Claus (1991): "Abstracts und Zusammenfassungen im deutsch-englischen Vergleich: Das Passiv als interkulturelles und teiltextdifferenzierendes Signal." In: Müller, Bernd-Dietrich (ed.): Interkulturelle Wirtschaftskommunikation. München, Iudicium: 363378.

Gnutzmann, Claus/Lange, Regina (1990): "Kontrastive Textlinguistik und Fachsprachenanalyse." In: Gnutzmann, Claus (ed.): Kontrastive Linguistik. Frankfurt a. M. u. a., Lang: 8516. (= Forum Angewandte Linguistik 19).

Göpferich, Susanne (1998): Textsorten in Naturwissenschaften und Technik. Pragmatische Typologie - Kontrastierung - Translation. Tübingen: Narr. (= Forum für Fachsprachenforschung 27).

Golebiowski, Zofia (2009): "Prominent Messages in Education and Applied Linguistic Abstracts: How do Authors Appeal to their Prospective Readers?". Journal of Pragmatics 41/4: 753-769.

Graetz, Naomi (1985): "Teaching EFL students to extract structural information from abstracts." In: Ulijn, J.M./Pugh, A.K. (eds.): Reading for professional purposes. Methods and materials in teaching languages. Leuven, Amersfoort: 123-135.

Haynes, Brian R. et al. (1990): "More informative abstracts revisited". Annals of Internal Medicine 113/1: 69-76.

Hutz, Matthias (1997): Kontrastive Fachtextlinguistik für den fachbezogenen Fremdsprachenunterricht. Fachzeitschriftenartikel der Psychologie im interlingualen Vergleich. Trier: Universitätsverlag.

Ickler, Theodor (1993): "Zur Textgattung Abstract". Fachsprache LSP Journal 15/1-2: 4453.

ISO 214-1976 (E) (1976): Documentation: Abstracts for publication and documentation. Genf.

Jordan, M. P. (1990): "The linguistic genre of abstracts". In: Volpe, Angela Della (ed.): The Seventeenth LACUS Forum 1990. Lake Bluff, Illinois: 507-527.

Kourilová, Magda (1996): "The non-native speaker and the skill of abstracting". UNESCO ALSED-LSP Newsletter Copenhagen 19/2: 12-21.

Kreisky, Eva (2005): "Anleitung zum wissenschaftlichen Arbeiten". http://evakreisky.at/wissenschaftlich_arbeiten/schreiben_zitieren.php, zitiert von http://mailbox.univie.ac.at/marion.loeffler/, Stand 31.1.2005.

Kretzenbacher, Heinz Leonhard (1990): Rekapitulation. Textstrategien der Zusammenfassung von wissenschaftlichen Fachtexten. Tübingen: Narr. (= Forum für Fachsprachenforschung 11).

Krvavac, Alka (1994): "Abstract as a text in science with respect to its information relevance". In: Brekke, Magnar et al. (eds.): Applications and implications of current LSP research. Bergen, Fagbokforlaget: 746-750.

KTS =Komitee Terminologie und Sprachfragen der Deutschen Gesellschaft für Dokumentation e.V. (ed.) (1975): Terminologie der Information und Dokumentation. München. (= DGD-Schriftenreihe 4).

Lenk, Hartmut P. (1994): Praktische Textsortenlehre. Helsinki: Finlandia.

Lorés-Sanz, Rosa (2008): "Genres in Contrast. The Exploration of Writers' Visibility in Research Articles and Research Article Abstracts." In: Burgess, Sally/Martín-Martín, Pedro (eds.): English as an Additional Language in Research Publication and Communication. Frankfurt a. M. et al., Lang: 105-122. (= Linguistic Insights: Studies in Language and Communication 61).

Lorés-Sanz, Rosa (2009): "Different Worlds, Different Audiences: A Contrastive Analysis of Research Article Abstracts." In: Suomela-Salmi, Eija/Dervin, Fred (eds.): Cross-Linguistic 
and Cross-Cultural Perspectives on Academic Discourse. Amsterdam/Philadelphia, Benjamins: 187-198. (= Pragmatics \& Beyond New Series 193)

Maher, John Christopher (1990): International medical communication. Edinburgh.

Martín-Martín, Pedro (2005): The Rhetoric of the Abstract in English and Spanish Scientific Discourse. A Cross-Cultural Genre-Analytic Approach. Frankfurt a. M. et al.: Peter Lang.

McIntosh, Neil (1996): "Abstract information and structure at scientific meetings". The Lancet 347: 544-545.

Melander, Björn et al. (1994): "Journal abstracts from three academic fields in the United States and Sweden: National and disciplinary proclivities?" In: Duszak, Anna (ed.): Intellectual styles and cross-cultural communication. Berlin, New York: de Gruyter.

Melander, Björn (1997): Journal abstracts from three academic fields in the United States and Sweden: national or disciplinary proclivities? Vortrag: Conference on Analysing LSPGenres, Aarhus 14.5-17.5.1997.

Müller, Ina (2008): Die Übersetzung von Abstracts aus translationswissenschaftlicher Sicht (Russisch-Deutsch-Englisch): eine Untersuchung am Beispiel von Abstracts aus russischen Fachzeitschriften zur Schweißtechnik und ihren Übersetzungen ins Deutsche und Englische. Berlin: Frank \& Timme.

Mulrow, Cynthia D. et al. (1988): "A proposal for more informative abstracts of review articles." Annals of Internal Medicine 108: 613-615.

Oldenburg, Antje (1991): Abstracts deutscher und englischer wissenschaftlicher Zeitschriftenaufsätze der Kommunikationsbereiche Maschinenbau und Pädagogik. Ein inter- und intralingualer Vergleich. Magisterarbeit 1991.

Oldenburg, Antje (1995): "Methodologische Grundlagen der kontrastiven Fachtextlinguistik". Fachsprache LSP Journal 17/3-4: 107-116.

Oldenburg, Hermann (1992): Angewandte Fachtextlinguistik. 'Conclusion' und Zusammenfassungen. Tübingen: Narr. (= Forum für Fachsprachenforschung 17).

Ruge, Marion (1992): "Abstracts aus Zeitschriften und Referateorganen der Mathematik, Physik und Chemie". In: Gläser, Rosemarie (ed.): Aktuelle Probleme der anglistischen Fachtextanalyse. Frankfurt a. M. et al.: Lang: 59-68. (= Leipziger Fachsprachen-Studien $5)$.

Tsai, Yvonne (2010): "Text Analysis of Patent Abstracts." JoSTrans 13: 61-80. http://www.jostrans.org/issue13/art_tsai.php, Stand 05.04.2010.

Salager-Meyer, Françoise (1990): "Discoursal movements in medical English abstracts and their linguistic exponents: A genre analysis study." Interface. Journal of Applied Linguistics 4.2: 107-124.

Santos, Mauro Bittencourt dos (1996): "The textual organization of research paper abstracts in applied linguistics." Text 16/ 4: 481-499.

Skern, Tim (2009): Writing Scientific English: A Workbook. Stuttgart, Wien: facultas wuv.

Swales, John M. (1990): Genre Analysis. English in academic and research settings. Cambridge et al.: CUP.

Swales, John M./Feak, Christine B. (1994): Academic writing for graduate students. Essential tasks and skills. Ann Arbor: The University of Michigan Press.

Tibbo, Helen R. (1992): "Abstracting across the disciplines: A content analysis of abstracts from the natural sciences, the social sciences, and the humanities with implications for abstracting standards and online information retrieval." LISR 14: 31-56.

Ventola, Eija (1994): "Abstracts as an object of linguistic study." In: Čmejrková, Svètla/Daneš, František/Havlová, Eva (eds.): Writing vs speaking. Language, text, discourse, communication. Tübingen, Narr: 333-352.

Webber, Pauline (1996): "Metaphor in medical English abstracts." UNESCO ALSED-LSP Newsletter 19/1: 35-52. 